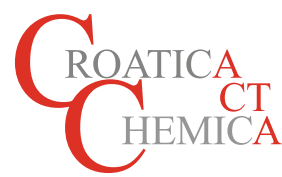

\title{
Some Less Familiar Properties of Randić Index
}

\author{
(1) Ivan Gutman
}

\author{
Faculty of Science, University of Kragujevac, P. O. Box 60, 34000 Kragujevac, Serbia \\ Author's e-mail address: gutman@kg.ac.rs \\ RECEIVED: February 13, 2021 * REVISED: April 1, 2021 * ACCEPTED: April 2, 2021
}

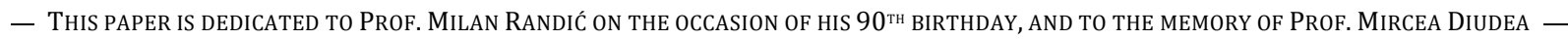

Abstract: Several mathematically relevant properties of the Randić connectivity index, that may be less familiar to the chemical community, are outlined and commented.

Keywords: Randić index, Randić matrix, Randić energy, Laplacian matrix, normalized Laplacian matrix, connectivity index.

\section{INTRODUCTION}

$\mathrm{N}$ his seminal and thousands-times quoted paper[1] "On characterization of molecular branching", Milan Randić introduced one of the most popular graph-based structure descriptors (topological indices). Randić himself named it "branching index", but the name was soon changed into "connectivity index". ${ }^{[2,3]}$ Eventually, the name "Randić index" (or, sometimes, "Randić connectivity index") has been accepted by the vast majority of contemporary scholars. It should be noted that "Randić index" is explicitly mentioned in the newest (2020) Mathematics Subject Classification, within the section 05 Combinatorics, subsection 05C Graph theory, and subject 05C09 Graphical indices; for details see. ${ }^{[4]}$

Let $G$ be a simple (molecular) graph with $n$ vertices and $m$ edges, whose vertex set is $V(G)=\left\{v_{1}, v_{2}, \ldots, v_{n}\right\}$ and edge set $E(G)$. The edge connecting the vertices $v_{i}$ and $v_{j}$ is denoted by $i j$. The degree (= number of first neighbors) of the vertex $v_{i}$ will be denoted by $d_{i}$.

In order to avoid trivialities, in what follows it is assumed that the graph $G$ is connected, possessing at least two vertices (and therefore at least one edge). Thus, $G$ has no isolated vertices, i.e., $d_{i} \geq 1$ for all $i=1,2, \ldots, n$.

For additional notions from chemical graph theory, the textbooks ${ }^{[5,6]}$ should be consulted.

In the above specified notation, the Randić index is defined as ${ }^{[1]}$

$$
R=R(G)=\sum_{i j \in E(G)} \frac{1}{\sqrt{d_{i} d_{j}}}
$$

It should be noted that the Randić index is one of the several vertex-degree-based molecular structure descriptors whose general form is

$$
T I=T I(G)=\sum_{i j \in E(G)} F\left(d_{i}, d_{j}\right)
$$

where $F(x, y)$ is a suitably chosen function whose property must be $F(x, y)=F(y, x)$. Nowadays, a few dozens of such topological indices are studied in the current mathematical and chemical literature. ${ }^{[7-11]}$

\section{GENERALIZATIONS OF RANDIĆ INDEX}

Since 1975, when the Randić index was introduced, its numerous generalizations were considered and investigated. The most straightforward is of the form

$$
R_{\alpha}=\sum_{i j \in E(G)}\left(d_{i} d_{j}\right)^{\alpha}
$$

for $\alpha$ being some real number. This expression is based on the fact that the ordinary Randić index, Eq. (1), pertains to $\alpha=-1 / 2 \cdot R_{\alpha}$ is usually referred to as the "general Randić index". ${ }^{[12,13]}$ The case $\alpha=-1$ attracted special attention of mathematicians. ${ }^{[14,15]}$

The expression on the right-hand side of Eq. (1) can be viewed as a connectivity index of the first order. If so, then the "second-order connectivity index" would be ${ }^{[2,3]}$ 


$$
{ }^{2} R=\sum_{i j k} \frac{1}{\sqrt{d_{i} d_{j} d_{k}}}
$$

where the summation goes over all 3-vertex path subgraphs of the underlying graph $G$. Analogously, the thirdorder index would be

$$
{ }^{3} R=\sum_{i j k \ell} \frac{1}{\sqrt{d_{i} d_{j} d_{k} d_{\ell}}}
$$

and similar for ${ }^{k} R, k=4,5, \ldots$. The zeroth-order Randić index and its "general" version

$$
{ }^{0} R=\sum_{v_{i} \in V(G)} \frac{1}{\sqrt{d_{i}}} \quad \text { and } \quad{ }^{0} R_{\alpha}=\sum_{v_{i} \in V(G)}\left(d_{i}\right)^{\alpha}
$$

in spite of their simplicity, have also been much investigated. ${ }^{[12,13]}$

In what follows, we focus our attention only to the mathematical properties of the ordinary Randić index, Eq. (1).

\section{AN IDENTITY FOR RANDIĆ INDEX}

Theorem 1. Let $G$ be a connected graph with $n$ vertices $v_{1}, v_{2}, \ldots, v_{n}$. Then its Randić index satisfies the relation

$$
R(G)=\frac{n}{2}-\sum_{i j \in E(G)}\left(\frac{1}{\sqrt{d_{i}}}-\frac{1}{\sqrt{d_{j}}}\right)^{2}
$$

The remarkable identity (2) was communicated in two papers, ${ }^{[16,17]}$ whereas its main chemical applications in the paper ${ }^{[18]}$ and elsewhere. ${ }^{[19]}$ From Theorem 1, the following is immediately evident:

Corollary 2. $R(G) \leq n / 2$.

Corollary 3. The equality $R(G)=n / 2$ holds for any regular graph with $n$ vertices. If the (molecular) graph $G$ is not regular, then $R(G)<n / 2$.

The results of Corollaries 2 and 3 were discovered in the 1980s, ${ }^{[19]}$ but were proven without the use of formula (2).

Corollary 3 has a noteworthy chemical consequence: in the case of regular graphs (such as the molecular graphs of fullerenes, nanotubes, and similar), the Randić index is completely insensitive to any structural detail (except the number of carbon atoms). Therefore, the applicability of Randić index is limited to (molecular) graphs in which the vertex degrees assume different values. Trees (molecular graphs of alkanes) are the best objects satisfying this condition. Indeed, the majority of applications of the Randić index is for (molecular) trees. The fundamental result along these lines is the following:

Theorem 4. Let $S_{n}$ and $P_{n}$ denote the $n$-vertex star and path (see Figure 1 ). Then for any $n$-vertex tree $T_{n}$, different from $S_{n}$ and $P_{n}$,

$$
R\left(P_{n}\right)<R\left(T_{n}\right)<R\left(S_{n}\right) .
$$

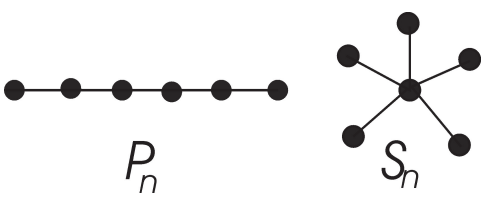

Figure 1. The path and the star graphs. Here their special cases for $n=6$ are depicted. Among trees with the same number of vertices, these have the smallest and the greatest Randić index.

Since, evidently, the path is the least branched and the star the most branched tree, inequalities (3) confirm that the Randić index is a proper measure of molecular branching.

In order to see how Theorem 4 can be deduced from Eq. (2), consider the term

$$
X_{i j}=\left(\frac{1}{\sqrt{d_{i}}}-\frac{1}{\sqrt{d_{j}}}\right)^{2}
$$

and note that in the case of trees, its maximal value is for $d_{i}=n-1, d_{j}=1$ or vice versa. All the $n-1$ edges of $S_{n}$ assume this maximal value. Therefore $S_{n}$ has the greatest Randić index among $n$-vertex trees.

A path has two pendent edges (= edges incident to a vertex of degree 1). Any other tree has 3 or more pendent edges.

The smallest value of $X_{i j}$ is for $d_{i}=d_{j}$, equal to zero. In the case of pendent edges, the second-smallest is $X_{i j}=1.5-\sqrt{2} \approx 0.09$ for $d_{i}=1, d_{j}=2$ or vice versa. The path $P_{n}$ has $n-3$ edges for which $X_{i j}=0$ and two (= the smallest possible number) with $X_{i j}=1.5-\sqrt{2}$. This evidently renders the smallest Randić index among $n$-vertex trees.

It is worth noting that the proof of the left-hand side of inequality (3), without using the identity (2), was the first non-trivial mathematical result obtained for the Randic index. ${ }^{[14]}$ The publication of the papers ${ }^{[14,15]}$ triggered intensive mathematical research of the Randić index and its generalizations, resulting in hundreds of publications and at least two books. ${ }^{[12,13]}$

\section{RANDIĆ MATRIX}

There are numerous matrices that can be associated with graphs. ${ }^{[21,22]}$ However, only two graph matrices have found extensive applications in both mathematics, computer science, and natural sciences. These are the adjacency matrix $A(G)$ and the Laplacian matrix $L(G)$. Both are symmetric square matrices of order $n$. Their $(i, j)$-elements are defined as

$$
A(G)_{i j}=\left\{\begin{array}{ll}
1 & \text { if } i j \in E(G) \\
0 & \text { if } i j \notin E(G) \\
0 & \text { if } i=j
\end{array} \text { and } \quad L(G)_{i j}=\left\{\begin{array}{cl}
-1 & \text { if } i j \in E(G) \\
0 & \text { if } i j \notin E(G) \\
d\left(v_{i}\right) & \text { if } i=j
\end{array}\right.\right.
$$


Details on the above defined graph matrices can be found in the monographs. ${ }^{[23,24]}$

For what follows, we need to define an auxiliary matrix $D=D(G)$ whose diagonal elements are the vertex degrees $d_{1}, d_{2}, \ldots, d_{n}$, whereas all off-diagonal elements are zero. Then the Laplacian and adjacency matrices are related as

$$
L(G)=D(G)-A(G)
$$

The normalized Laplacian matrix is defined as ${ }^{[25]}$

$$
L_{\text {norm }}(G)=D^{-1 / 2} L(G) D^{-1 / 2}
$$

which by (4) becomes

$$
L_{\text {norm }}(G)=I_{n}-D^{-1 / 2} A(G) D^{-1 / 2}
$$

where $I_{n}$ stands for the unit matrix of order $n$.

By direct calculation we find that the $(i, j)$-element of the matrix $D^{-1 / 2} A(G) D^{-1 / 2}$ is equal to:

$$
\left[D^{-1 / 2} A(G) D^{-1 / 2}\right]_{i j}= \begin{cases}1 / \sqrt{d\left(v_{i}\right) d\left(v_{j}\right)} & \text { if ij } \in E(G) \\ 0 & \text { if ij } \notin E(G) \\ 0 & \text { if } i=j\end{cases}
$$

Bearing in mind Eq. (1), we arrive at the following definitions. ${ }^{[26,27]}$

Definition 5. The matrix whose $(i, j)$-element is given by Eq. (6) is the Randic matrix of the underlying graph $G$ and will be denoted by $R M=R M(G)$.

Thus, $R M(G)$ is a symmetric square matrix of order $n$, with zero diagonal, whose sum of all elements is equal to twice the Randić index of the graph $G$.

Definition 6. The eigenvalues of the Randić matrix, denoted by $\rho_{1}, \rho_{2}, \ldots, \rho_{n}$, are the Randić eigenvalues of the graph $G$. They form the Randić spectrum of the graph $G$. The greatest Randić eigenvalue is said to be the Randić spectral radius of the graph $G$.

The Randić eigenvalues are real-valued numbers, whose sum is equal to zero.

Definition 7. The sum of the absolute values of the Randić eigenvalues, denoted by $R E=R E(G)$, i.e.,

$$
R E(G)=\sum_{i=1}^{n}\left|\rho_{i}\right|
$$

is the Randić energy of the graph $G$.

The above Definitions 5-7 were first time stated in the papers, ${ }^{[26,27]}$ and were introduced knowing Eq. (5). It is worth noting that the paper ${ }^{[2]]}$ was dedicated to Milan Randić, on the occasion of his $80^{\text {th }}$ birthday.
By recognizing Eq. (5), it became evident that the concept of Randić matrix (and, indirectly, of the Randić index) has a deep-lying algebraic root, and is connected with the theory of Laplacian matrices.

Since 2010, when the papers ${ }^{[26,27]}$ were published, the concept of Randić matrix, and especially of its spectrum and energy, attracted much attention and were studied in scores of publications. Some of these are surveyed in the subsequent section.

\section{RANDIĆ ENERGY}

If the eigenvalues of the adjacency matrix $A(G)$ are $\lambda_{1}, \lambda_{2}, \ldots, \lambda_{n}$, then the energy of the graph $G$ is defined as ${ }^{[28]}$

$$
G E=G E(G)=\sum_{i=1}^{n}\left|\lambda_{i}\right|
$$

The relation between graph energy and the total $\pi$-electron energy is described in detail elsewhere. ${ }^{[28,29]}$ The graph energy showed to be a significant and fruitful mathematical concept and its research resulted in hundreds of results and publications. ${ }^{[30,31]}$ This success was a motivation to introduce energies of other matrices. ${ }^{[29]}$ One among these is the Randić energy, Eq. (7), Definition 7. Evidently, Eq. (7) is constructed in full analogy with Eq. (8).

In the same year (2010) when the Randić energy was introduced, ${ }^{[26,27]}$ Cavers et al. ${ }^{[32]}$ put forward the concept of normalized Laplacian energy, namely the energy of the normalized Laplacian matrix $L_{\text {norm }}(G)$. Let $\mu_{1}, \mu_{2}, \ldots, \mu_{n}$ be the eigenvalues of $L_{\text {norm }}(G)$. Then, in view of $\mu_{1}+\mu_{2}+\cdots+\mu_{n}=n$, the respective normalized Laplacian energy is defined as

$$
N L E(G)=\sum_{i=1}^{n}\left|\frac{1}{n}-\mu_{i}\right| .
$$

It was not long to recognize that the two energies coincide. ${ }^{[26]}$

Theorem 8. For any connected graph $G$, the normalized Laplacian matrix and the Randic matrix are related as $L_{\text {norm }}(G)=I_{n}-R M(G)$. Therefore, $\mu_{i}=1-\rho_{i}, i=1,2, \ldots, n$, holds.

Corollary 9. The normalized Laplacian and Randić energies coincide, $\operatorname{NLE}(G)=\operatorname{RE}(G)$.

In the literature that followed, there were a few publications concerned with the normalized Laplacian energy, e.g. the recent papers, ${ }^{[33-35]}$ but the majority of them use the name "Randic energy". According to our census, ${ }^{[30,31]}$ by 2020 , there are 30 publications whose titles mention Randić energy, plus a review. ${ }^{[36]}$

The fundamental result on Randić energy, analogous to Theorem 4 is: ${ }^{[32,37,38]}$ 
Claim 10. (a) The $n$-vertex tree with the smallest Randic energy is the sun-graph (if $n$ is odd) and the balanced double sun (if $n$ is even), see Figure 2 .

(b) The $n$-vertex tree with the greatest Randic energy is the star, see Figure 1 . This is also the $n$-vertex graph with greatest Randić energy.

Claim 10(b) was proven by Cavers et al. ${ }^{[32]}$ Claim 10(a) was established in ${ }^{[37,38]}$ by means of computer search, but its rigorous mathematical proof is still missing.

Most papers dealing with Randić energy report lower and upper bounds and/or establish (or conjecture) the extremal cases. A characteristic result of this kind is the following: ${ }^{[39]}$

Theorem 11. Let $G$ be a connected graph with $n$ vertices. Let $R_{-1}$ be the general Randić index for $\alpha=-1$. Then

$$
\begin{aligned}
& 1+\sqrt{(n-1)(n-2)|\operatorname{det}(R M)|^{2 /\{n-1)}+2 R_{-1}-1} \leq R E(G) \leq \\
& 1+\sqrt{(n-1)\left(2 R_{-1}-1\right)} .
\end{aligned}
$$

If the maximum degree of $G$ is equal to $n-1$, then both equalities hold if and only if

$$
G \cong K_{n} \text { or } G \cong r K_{2} \cup K_{1}, n=2 r+1, r \geq 2 .
$$

Note that the determinants of the Randic and adjacency matrices are related as

$$
\operatorname{det} R M(G)=\frac{\operatorname{det} A(G)}{d_{1} d_{2} \cdots d_{n}}
$$

and recall that the structure-dependency of $\operatorname{det} A(G)$ of molecular graphs is studied in full detail. ${ }^{[40]}$

In a few papers, ${ }^{[41,42]}$ Randić energies of special graphs were computed.

Two non-isomorphic graphs having equal Randić energies are said to be Randić -equienergetic. Constructions of Randić-equienergetic graphs (with equal number of vertices) were reported in. ${ }^{[43,44]}$

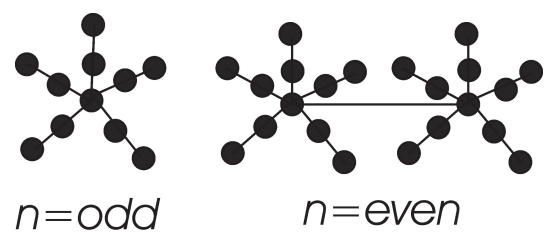

Figure 2. The trees whose Randic energy is minimal. Here the sun-graph with $n=11$ vertices and the balanced double sun with $n=22$ vertices are depicted. Note that these differ very much from the path (Figure 1), implying that the Randić energy is not a measure of molecular branching. In the case of even $n$, the degrees of the two central vertices of the double sun are either equal or differ by one.
There were numerous attempts to extend and generalize the Randić-energy concept. The most straightforward was to conceive a "general Randić energy", [45] containing a variable exponent $\alpha$. Of the variety of modified Randić-type energies, we mention here the following:

$$
\begin{aligned}
& \text { Hermite-Randić energy }{ }^{[46]} \\
& \text { Randić incidence energy }{ }^{[47]} \\
& \text { minimum dominating Randić energy }{ }^{[48]} \\
& \text { minimum equitable dominating Randić energy }{ }^{[49,50]} \\
& \text { Randić color energy } y^{[51]} \\
& \text { minimum covering Randić energy } y^{[52,53]} \\
& \text { Laplacian minimum covering Randić energy }{ }^{[54]} \\
& \text { skew Randić energy } \\
& \text { Randić-type additive connectivity energy }
\end{aligned}
$$

\section{RANDIĆ INDEX AND GRAPH ENERGY}

In two recently published papers, ${ }^{[57,58]}$ remarkably simple inequalities between the Randić index, Eq. (1), and graph energy, Eq. (8), were established.

Theorem 12. Let $G$ be a connected graph whose maximal vertex degree is $\Delta$. Then ${ }^{[57,58]}$

$$
\frac{1}{2 \Delta} G E(G) \leq R(G) \leq \frac{1}{2} G E(G) .
$$

Equality on the left-hand side is attained only if $G \cong P_{2}$. Equality on the right-hand side is attained only for $G \cong K_{a, b}$, a complete bipartite graph.

The left inequality was discovered by Yan et al. ${ }^{[57]}$ whereas the right inequality by Gerardo and Octavio Arizmendi. ${ }^{[58]}$ In spite of their simplicity, proving the inequalities (9) is a very difficult mathematical task. Namely, whereas $R(G)$ is a vertex-degree-dependent quantity, $G E(G)$ depends on graph eigenvalues. Thus, (9) connect two mathematically fully unrelated structure descriptors.

From the inequalities (9), one could expect that Randić index and graph energy are linearly correlated. This, however, is not the case. Numerical testing on trees and benzenoid systems revealed that the relation between $R(G)$ and $G E(G)$ is much more complex than linear. The most convincing example for the absence of any correlation between $R(G)$ and $G E(G)$ are regular graphs (e.g., molecular graphs of fullerenes). For such graphs, according to Corollary 3, the Randić index is a constant, independent of any variation in (molecular) structure. In contrast to this, graph energy of regular graphs has a pronounced sensitivity to their structure. $[28,59,60]$

\section{CONCLUDING REMARKS}

By reading Milan Randić's original paper, ${ }^{[1]}$ our impression is that the author was not aware of the deep-lying mathematical roots of his "branching index". Such fortunate 
mathematical consequences of "naïve" chemistry-motivated ideas happened not once in the history of theoretical chemistry.

Anyway, it was our privilege to collect the various mathematical results related to, or originating from, the concept of "branching index" invented by Milan Randić. Let its author be proud and happy, and continue to his $100^{\text {th }}$ birthday.

\section{REFERENCES}

[1] M. Randić, J. Am. Chem. Soc. 1975, 97, 6609-6615. https://doi.org/10.1021/ja00856a001

[2] L. B. Kier, L. H. Hall, Molecular Connectivity in Chemistry and Drug Research, Academic Press, New York, 1976.

[3] L. B. Kier, L. H. Hall, Molecular Connectivity in Structure-Activity Analysis, Wiley, New York, 1986.

[4] I. Gutman, MATCH Commun. Math. Comput. Chem. 2020, 84, 3.

[5] N. Trinajstić, Chemical Graph Theory, CRC Press, Boca Raton, 1983.

[6] S. Wagner, H. Wang, Introduction to Chemical Graph Theory, CRC Press, Boca Raton, 2018. https://doi.org/10.1201/9780429450532

[7] R. Todeschini, V. Consonni, Molecular Descriptors for Chemoinformatics, Wiley-VCH, Weinheim, 2009. https://doi.org/10.1002/9783527628766

[8] V. R. Kulli, in: M. Pal, S. Samanta, A. Pal (Eds.), Handbook of Research of Advanced Applications of Graph Theory in Modern Society, Global, Hershey, 2020, pp. 66-91.

[9] A. Martinez-Perez, J. M. Rodriguez, MATCH Commun. Math. Comput. Chem. 2019, 82, 679-698.

[10] I. Tomescu, MATCH Commun. Math. Comput. Chem. 2021, 85, 285-294.

[11] I. Gutman, MATCH Commun. Math. Comput. Chem. 2021, 86, 11-16.

[12] X. Li, I. Gutman, Mathematical Aspects of RandićType Molecular Structure Descriptors, Univ. Kragujevac, Kragujevac, 2006.

[13] I. Gutman, B. Furtula (Eds.), Recent Results in the Theory of Randić Index, Univ. Kragujevac, Kragujevac, 2008.

[14] B. Bollobás, P. Erdős, Ars Combin. 1998, 50, 225238.

[15] B. Bollobás, P. Erdős, A. Sarkar, Discrete Math. 1999, 200, 5-19.

https://doi.org/10.1016/\$0012-365X(98)00320-3

[16] I. Gutman, O. Araujo, J. Rada, Acta Chim. Hung. Models Chem. 2000, 137, 653-658.

[17] L. Pavlović, I. Gutman, Novi Sad J. Math. 2001, 31, 53-58.
[18] G. Caporossi, I. Gutman, P. Hansen, L. Pavlović, Comput. Biol. Chem. 2003, 27, 85-90. https://doi.org/10.1016/S0097-8485(02)00016-5

[19] I. Gutman, Selected Theorems in Chemical Graph Theory, Univ. Kragujevac, Kragujevac, 2017.

[20] S. Fajtlowicz, Congr. Numer. 1987, 60, 187-197.

[21] D. Janežič, A. Miličević, S. Nikolić, N. Trinajstić, Graph-Theoretical Matrices in Chemistry, Univ. Kragujevac, Kragujevac, 2007.

[22] D. Janežič, A. Miličević, S. Nikolić, N. Trinajstić, Graph-Theoretical Matrices in Chemistry, CRC Press, Boca Raton, 2015. https://doi.org/10.1201/b18389

[23] N. Biggs, Algebraic Graph Theory, Cambridge Univ. Press, Cambridge, 1993.

[24] C. D. Godsil, G. Royle, Algebraic Graph Theory, Springer, New York, 2001. https://doi.org/10.1007/978-1-4613-0163-9

[25] R. K. Fan Chung, Spectral Graph Theory, Amer. Math. Soc., Providence, 1997.

[26] S. B. Bozkurt, A. D. Güngör, I. Gutman, A. S. Cevik, MATCH Commun. Math. Comput. Chem. 2010, 64, 239-250.

[27] S. B. Bozkurt, A. D. Güngör, I. Gutman, $\mathrm{MATCH}$ Commun. Math. Comput. Chem. 2010, 64, 321-334.

[28] X. Li, Y. Shi, I. Gutman, Graph Energy, Springer, New York, 2012.

https://doi.org/10.1007/978-1-4614-4220-2

[29] I. Gutman, B. Furtula, Croat. Chem. Acta 2017, 90, 359-368. https://doi.org/10.5562/cca3189

[30] I. Gutman, B. Furtula, Energies of Graphs - Survey, Census, Bibliography, Center Sci. Research, Kragujevac, 2019.

[31] I. Gutman, H. Ramane, MATCH Commun. Math. Comput. Chem. 2020, 84, 277-292.

[32] M. Cavers, S. Fallat, S. Kirkland, Linear Algebra Appl. 2010, 433, 172-190.

[33] F. Tura, MATCH Commun. Math. Comput. Chem. 2017, 77, 617-624.

[34] G. P. Clemente, A. Cornaro, MATCH Commun. Math. Comput. Chem. 2017, 77, 673-690.

[35] A. D. Maden, MATCH Commun. Math. Comput. Chem. 2018, 79, 321-330.

[36] K. C. Das, S. Sorgun, K. Xu, in: I. Gutman, X. Li (Eds.), Graph Energies - Theory and Applications, Univ. Kragujevac, Kragujevac, 2016, pp. 111-122.

[37] B. Furtula, I. Gutman, Maced. J. Chem. Chem. Eng. 2013, 32, 117-123.

https://doi.org/10.20450/mjcce.2013.127

[38] I. Gutman, B. Furtula, S. B. Bozkurt, Linear Algebra Appl. 2014, 442, 50-57.

https://doi.org/10.1016/j.laa.2013.06.010

[39] K. C. Das, S. Sun, MATCH Commun. Math. Comput. Chem. 2017, 77, 77-84. 
[40] A. Graovac, I. Gutman, Croat. Chem. Acta 1978, 51, 133-140.

[41] S. Alikhani, N. Ghanbari, Appl. Math. Comput. 2015, 269, 722-730.

[42] S. Alikhani, N. Ghanbari, J. Math. Extension 2015, 9 , 73-85.

[43] O. Rojo, L. Medina, MATCH Commun. Math. Comput. Chem. 2012, 68, 805-814.

[44] S. Sorgun, H. Kücük, N. Kartal, MATCH Commun. Math.Comput. Chem. 2019, 81, 443-452.

[45] R. Gu, F. Huang, X. Li, Trans. Combin. 2014, 3, 21-33. https://doi.org/10.22108/toc.2014.5451

[46] Y. Lu, L. Wang, Q. Zhou, J. Ineq. Appl.2017, 54. https://doi.org/10.1186/s13660-017-1329-8

[47] R. Gu, F. Huang, X. Li, Trans. Comb. 2014, 3, 1-9. https://doi.org/10.22108/toc.2014.5573

[48] P. S. K. Reddy, K. N. Prakasha, V. M. Siddalingaswamy, Vladikavkaz. Math. J. 2017, 19, 28-35 https://doi.org/10.23671/VNC.2017.2.6506

[49] P. S. K. Reddy, K. N. Praksha, K. Gavirangiah, Int. J. Math. Combin. 2017, 3, 81-89. https://doi.org/10.5281/zenodo.1419027

[50] P. Rajendra, R. Rangarajan, Int. J. Math. Combin. 2018, 1, 97-108. https://doi.org/10.5281/zenodo.1419017
[51] P. Rajendra, Palestine J. Math. 2019, 8, 207-212.

[52] M. R. Rajesh Kanna, R. Jagadeesh, Adv. Linear Algebra Matrix Theory 2016, 6, 116-131.

[53] K. N. Prakasha, S. K. R. Polaepalli, I. N. Cangul, Kyungpook Math. J. 2017, 57, 701-709. https://doi.org/10.5666/kmj.2017.57.4.701

[54] M. R. Rajesh Kanna, R. Jagadeesh, Commun. Math. Appl. 2018, 9, 167-188.

[55] R. Gu, F. Huang, X. Li, Trans. Combin. 2016, 5(1), 1-14. https://dx.doi.org/10.22108/toc.2016.9513

[56] K. V. Madhusudhan, P. S. K. Reddy, K. R. Rajanna, Vladikavkaz Math. J. 2019, 21, 18-26. https://dx.doi.org/10.23671/VNC.2019.2.32113

[57] Z.Yan, C. Liu, Y. Pan, J. Li, MATCH Commun. Math. Comput. Chem. 2021, 86, 539-542.

[58] G. Arizmendi, O. Arizmendi, Linear Algebra Appl. 2021, 609, 332-338. https://doi.org/10.1016/j.laa.2020.09.025

[59] A. Aashtab, S. Akbari, E. Ghasemian, A. H. Ghodrati, M. A. Hosseinzadeh, F. Koorepazan-Moftakhar, Linear Algebra Appl. 2019, 581, 51-57. https://doi.org/10.1016/j.laa.2019.07.001

[60] I. M. Jovanović, E. Zogić, MATCH Commun. Math. Comput. Chem. 2021, 86, 559-575. 